\title{
Treatment patterns of chronic obstructive pulmonary disease in employed adults in the United States
}

This article was published in the following Dove Press journal:

International Journal of COPD

24 February 2015

Number of times this article has been viewed

\author{
Gregory B Diette' \\ Anand A Dalal ${ }^{2}$ \\ Anna O D'Souza ${ }^{3}$ \\ Orsolya E Lunacsek ${ }^{3}$ \\ Saurabh P Nagar ${ }^{2}$
}

'Johns Hopkins University, Baltimore, MD, ${ }^{2}$ GlaxoSmithKline, Research Triangle Park, NC, ${ }^{3}$ Xcenda, Palm Harbor, FL, USA
Correspondence: Orsolya E Lunacsek Xcenda, LLC, 4 II 4 Woodlands Parkway, Suite 500, Palm Harbor, FL 34685, USA

$\mathrm{Tel}+\mathrm{I} 727$ 77| 4100

Fax + I 727 77| 4|45

Email orsolya.lunacsek@xcenda.com
Background: This study evaluated patterns of pharmacotherapy in chronic obstructive pulmonary disease (COPD) as they relate to recommended guidelines in a prevalent COPD patient population with employer-sponsored health insurance in the US.

Methods: Health care claims data from 2007 and 2008 were retrospectively analyzed for the study population defined as patients aged 40 years and older, continuously enrolled during the study period, and having at least one inpatient or one emergency department (ED) visit, or at least two outpatient claims coded with COPD (International Classification of Diseases, 9th Revision, Clinical Modification code 491.xx, 492.xx, 496.xx). Rates of any pharmacotherapy (both maintenance and reliever), long-acting maintenance pharmacotherapy in patients with an exacerbation history, and short-term treatment of acute exacerbations of COPD were evaluated in the overall population, newly diagnosed, and previously diagnosed patients (including maintenance-naïve and maintenance-experienced). Stratified analyses were also conducted by age group (40-64 years, $\geq 65$ years) and physician specialty.

Results: A total of 55,361 patients met study criteria of whom $39 \%$ were newly diagnosed. The mean age was 66 years, and $46 \%$ were male. Three-fourths (74\%) of all COPD patients had some pharmacotherapy (maintenance or reliever) with less than half (45\%) being treated with maintenance medications. The combination of an inhaled corticosteroid and a long-acting beta-agonist was the most prevalent drug class for maintenance treatment followed by tiotropium. Only $64 \%$ of patients with an exacerbation history had a prescription for a long-acting maintenance medication, and short-term treatment with oral corticosteroids or antibiotics was higher for hospitalization exacerbations compared to ED visit exacerbations (68\% vs 44\%). In general, the rates of pharmacotherapy were highest in patients who were maintenanceexperienced followed by newly diagnosed and maintenance-naïve.

Conclusion: The majority of COPD patients received maintenance or reliever COPD medications, but less than half received guideline-recommended care, especially those with an exacerbation history or receiving short-term treatment for acute exacerbations.

Keywords: COPD, pharmacotherapy, maintenance treatment, exacerbations

\section{Introduction}

Chronic obstructive pulmonary disease (COPD) is a chronic respiratory disorder with significant morbidity and mortality, and is the fourth leading cause of death worldwide. ${ }^{1}$ In the US, it affects more than 12 million people and is the third leading cause of death. ${ }^{2,3}$ COPD also imposes a significant economic burden accounting for an estimated $\$ 49.9$ billion of the US health care budget annually ( $\$ 29.5$ billion direct and $\$ 20.4$ billion indirect costs), and $€ 38.6$ billion annually in the European Union. ${ }^{1}$ 
COPD is a progressive and symptomatic disease characterized primarily by shortness of breath, cough, and sputum production. ${ }^{1}$ Along with smoking cessation, which is the only treatment modality known to significantly limit disease progression, ${ }^{4,5}$ pharmacologic therapy is central to the management of COPD patients. ${ }^{1}$ Treatment with drugs is primarily indicated for symptomatic relief, for prevention and treatment of exacerbations, and for improving health status. The Global initiative for chronic Obstructive Lung Disease (GOLD) has developed guidelines for various aspects of COPD management including pharmacotherapy. ${ }^{1}$ These guidelines are updated regularly based on new evidence from clinical trials and observational research. ${ }^{1}$

A few studies have evaluated the patterns of drug therapy use relative to the GOLD guidelines, and have identified several gaps in pharmacological management of COPD. ${ }^{6-8}$ Most of these studies used data before 2007. Since then, several pivotal trials demonstrating significant benefits of using maintenance drugs for COPD management have been published, such as the Towards a Revolution in COPD Health (TORCH) and the Understanding Potential Longterm Impacts on Function with Tiotropium (UPLIFT). ${ }^{9,10}$ Additionally, prior studies have not examined the treatment patterns and drug therapy uptake in COPD patients who were newly diagnosed or those without prior maintenance treatment. Therefore, an updated evaluation of current treatment patterns is required to assess improvement, if any, in the pharmacological management of COPD from the US perspective.

This study evaluated patterns of pharmacotherapy as they relate to recommended guidelines in a large COPD patient population using administrative claims data. The intent was to evaluate a prevalent COPD population including both newly diagnosed and previously diagnosed patients. However, there might be differences in treatment patterns between these two subgroups of patients, and furthermore between previously diagnosed patients who are treated vs those who are not treated with maintenance pharmacotherapy. Therefore, the study analyzed patterns of pharmacotherapy based on prior diagnosis as well as prior maintenance treatment.

\section{Methods}

A cross-sectional study design was employed using data from January 1, 2007 to December 31, 2008 (ie, the study period). The year 2008 was the analytic year of interest, termed the index year, during which the patterns and prevalence of pharmacotherapy were evaluated, while 2007, termed as the pre-index year, was used to assess baseline characteristics and to ascertain previous diagnosis of COPD. Pharmacotherapy for COPD consisted of maintenance and reliever medications. Maintenance medications included inhaled corticosteroid (ICS), long-acting beta-agonist (LABA), fixed-dose combination ICS + LABA, tiotropium (TIO), and inhaled ipratropium or fixed-dose combination ipratropium + albuterol (collectively referred to as ipratropium [IPR]). Reliever medications included short-acting beta-agonist (SABA), oral corticosteroid (OCS), nebulized ipratropium or combination ipratropium-albuterol (collectively referred to as nebulized ipratropium [nebIPR]), and antibiotics.

Patients $\geq 40$ years of age were identified during the index year (2008) as being diagnosed with COPD (International Classification of Diseases, 9th Revision, Clinical Modification [ICD-9 CM] code 491.xx, 492.xx, or 496.xx in primary or secondary fields) if they had 1) at least one hospitalization with COPD as the discharge diagnosis, or 2) at least one emergency department (ED) visit with a COPD diagnosis, or 3) at least two visits in any other setting on different dates with a COPD diagnosis. All patients were required to have continuous eligibility during the study period and be not diagnosed with other major respiratory conditions that would likely skew treatment patterns representative of an average COPD patient (pulmonary tuberculosis, sarcoidosis, respiratory cancer, cystic fibrosis, bronchiectasis, extrinsic allergic alveolitis, pneumonociosis and other lung diseases due to external agents, empyema, pleurisy, pneumothorax, abscess of lung and mediastinum, pulmonary congestion and hypostasis, pulmonary fibrosis, other alveolar and parietoalveolar pneumonopathy, lung involvement in conditions classified elsewhere, pulmonary collapse, interstitial and compensatory emphysema, pulmonary eosinophilia, acute edema of lung, unspecified, pulmonary insufficiency following trauma and surgery, allergic bronchopulmonary aspergillosis, transfusionrelated acute lung injury, chronic respiratory failure, and other disease of the respiratory system).

The pre-index year (2007) was used to classify the eligible COPD patients as newly diagnosed or previously diagnosed with COPD. Specifically, patients were categorized as newly diagnosed COPD patients if they did not have a medical claim with a COPD diagnosis and did not use ipratropium or any other maintenance drug for COPD in 2007. Patients were considered previously diagnosed if they had a medical claim with a COPD diagnosis in 2007. Previously diagnosed COPD patients were further categorized as maintenanceexperienced (ME) or maintenance-naïve (MN) if they did or did not have evidence of use of maintenance medications for COPD in 2007, respectively. 


\section{Data source}

The Truven Health MarketScan ${ }^{\circledR}$ Commercial Claims and Encounters and the Medicare Supplemental Databases were used for this study. The Commercial Claims and Encounters Database includes 34 million lives per year and represents the medical experience of active employees, early retirees, Consolidated Omnibus Budget Reconciliation Act (COBRA) continuees, and their dependents insured by employer-sponsored plans (ie, non-Medicare eligibles). The Medicare Supplemental Database includes three million lives per year and represents Medicare-eligible active and retired employees and their Medicare-eligible dependents from employer-sponsored supplemental plans. Both databases have medical and pharmacy claims linked to patient enrollment data containing demographic and health insurance plan characteristics. The medical service claims contain detailed information on both inpatient and outpatient care, such as date and place of service, payments, and up to 15 ICD-9 $\mathrm{CM}$ diagnosis and procedure codes. The pharmacy claims provide details from all prescriptions dispensed including the National Drug Code, dispense date, quantity dispensed, days supplied, and plan- and patient-paid amounts.

\section{Outcomes}

All outcomes were evaluated in the index year (2008). The study assessed the prevalence of any kind of drug treatment for COPD symptom management defined as the proportion of all COPD patients with at least one prescription claim for a maintenance or reliever medication. Further detail about pharmacotherapy patterns was provided separately for maintenance therapy and reliever therapy, as well as specific medication classes within these two categories. The study also examined real-world adherence to specific guideline-recommended care, including the prevalence of long-acting maintenance pharmacotherapy (ie, ICS + LABA, ICS, LABA, TIO) in patients with prior exacerbations, and the prevalence of OCS or antibiotic use for short-term treatment (within 5 days of discharge) of an acute exacerbation of COPD (hospitalization or ED visit). ${ }^{11}$ A COPD-related exacerbation was defined as a COPD-related 1) hospitalization, or 2) ED visit, or 3) physician/outpatient visit accompanied by a prescription for an OCS or an antibiotic within 5 days of that visit. ${ }^{11}$ A COPD-related visit (hospitalization/ ED visit/physician visit/outpatient visit) was defined as a visit with a primary discharge diagnosis of COPD, or with a primary discharge diagnosis of a qualifying respiratory condition (acute bronchitis and bronchiolitis, pneumonia and influenza, acute respiratory failure, other pulmonary insufficiency, acute and chronic respiratory failure, dyspnea, and respiratory abnormalities) with a secondary discharge diagnosis of COPD.

\section{Analytical approach}

Descriptive statistics were used to report the outcomes for the overall and the subgroup populations (newly diagnosed, ME, $\mathrm{MN})$. Furthermore, stratified analyses by age (40-64 years and $\geq 65$ years) and physician specialty were conducted. Patients were classified as being treated by a pulmonologist or general practitioner (GP) based on specialty information coded on medical claims during the index year. If a pulmonologist was identified on at least one medical claim (any diagnosis), the patient was considered to be under the care of a pulmonologist. For the remaining patients, if a GP was identified on at least one medical claim, the patient was considered to be under the care of a GP. Patients who could not be classified to pulmonology or GP were excluded from the specialty-level analyses. The baseline description of the study sample was based on variables from the preindex year (2007) including age, sex, geographic region, pre-index medications, pre-index medication dispensing, COPD reliever medication use (SABA, OCS, nebIPR), Charlson comorbidity index (CCI) score, number of unique diagnoses, and the presence of asthma, depression, upper respiratory tract infection, lower respiratory tract infection, and cardiovascular disease.

\section{Results}

A total of 55,361 patients met study criteria, of whom 21,314 patients $(38.5 \%)$ had newly diagnosed COPD. Among previously diagnosed patients, approximately half $(54 \%)$ were categorized as ME and $46 \%$ as MN patients. The mean age of the study population was 66 years, where newly diagnosed patients were younger than previously diagnosed patients, and ME patients tended to be older than 65 years (Table 1). Previously diagnosed patients had the highest comorbidity burden as shown by the higher CCI scores and increased prevalence of other assessed comorbid conditions. ME patients had the highest utilization of reliever medications at baseline. Less than $12 \%$ of newly diagnosed patients were using SABAs or OCSs during the pre-index year.

Of the full study sample, 48,573 patients (88\%) were identified for the specialty-level stratified analyses. In the index year, $25 \%$ of these patients were under the care of a pulmonologist (23\%, $25 \%$, and $55 \%$ for newly diagnosed, $\mathrm{MN}$, and ME patients, respectively), and the remainder were classified as under the care of a GP. 
Table I Demographic and clinical description of study sample during pre-index year

\begin{tabular}{|c|c|c|c|c|c|c|c|c|}
\hline \multirow[t]{3}{*}{ Characteristics } & \multirow{2}{*}{\multicolumn{2}{|c|}{ Total }} & \multirow{2}{*}{\multicolumn{2}{|c|}{ Newly diagnosed }} & \multicolumn{4}{|c|}{ Previously diagnosed } \\
\hline & & & & & \multirow{2}{*}{\multicolumn{2}{|c|}{$\begin{array}{l}\text { Maintenance- } \\
\text { naïve } \\
\mathrm{N}=15,688\end{array}$}} & \multirow{2}{*}{\multicolumn{2}{|c|}{$\begin{array}{l}\text { Maintenance- } \\
\text { experienced } \\
\mathrm{N}=18,359\end{array}$}} \\
\hline & \multicolumn{2}{|c|}{$N=55,36 I$} & \multicolumn{2}{|c|}{$\mathbf{N}=2|, 3| 4$} & & & & \\
\hline \multicolumn{9}{|l|}{ Demographic } \\
\hline Age in years (mean, SD) & 66.25 & 12.3 & 63.30 & 12.6 & 65.89 & 12.3 & 69.99 & 10.7 \\
\hline \multicolumn{9}{|l|}{ Age group in years $(n, \%)$} \\
\hline $40-64$ & 27,986 & $50.6 \%$ & 13,350 & $62.6 \%$ & 8,577 & $54.7 \%$ & 6,059 & $33.0 \%$ \\
\hline$\geq 65$ & 27,375 & $49.4 \%$ & 7,964 & $37.4 \%$ & 7,111 & $45.3 \%$ & 12,300 & $67.0 \%$ \\
\hline Female (n, \%) & 29,829 & $53.9 \%$ & $\mid 1,513$ & $54.0 \%$ & 8,551 & $54.5 \%$ & 9,765 & $53.2 \%$ \\
\hline \multicolumn{9}{|l|}{ Region (n, \%) } \\
\hline Northeast & 4,851 & $8.8 \%$ & 1,990 & $9.3 \%$ & $\mathrm{I}, 243$ & $7.9 \%$ & 1,618 & $8.8 \%$ \\
\hline North Central & 20,010 & $36.1 \%$ & 7,176 & $33.7 \%$ & 5,409 & $34.5 \%$ & 7,425 & $40.4 \%$ \\
\hline South & 23,231 & $42.0 \%$ & 9,441 & $44.3 \%$ & 7,189 & $45.8 \%$ & 6,601 & $36.0 \%$ \\
\hline West & 7,193 & $13.0 \%$ & $2,67 \mid$ & $12.5 \%$ & 1,825 & $11.6 \%$ & 2,697 & $14.7 \%$ \\
\hline Unknown & 76 & $0.1 \%$ & 36 & $0.2 \%$ & 22 & $0.1 \%$ & 18 & $0.1 \%$ \\
\hline \multicolumn{9}{|l|}{ Overall disease burden } \\
\hline $\mathrm{CCl}$ (mean, SD) & 1.00 & 1.3 & 0.74 & 1.2 & 1.16 & 1.4 & 1.17 & 1.4 \\
\hline Asthma (n, \%) & 8,014 & $14.5 \%$ & 1,449 & $6.8 \%$ & 2,480 & $15.8 \%$ & 4,085 & $22.3 \%$ \\
\hline URTI (n, \%) & 9,852 & $17.8 \%$ & 3,838 & $18.0 \%$ & 3,072 & $19.6 \%$ & 2,942 & $16.0 \%$ \\
\hline LRTI (n, \%) & 11,184 & $20.2 \%$ & 2,879 & $13.5 \%$ & 3,643 & $23.2 \%$ & 4,662 & $25.4 \%$ \\
\hline CVD (n, \%) & 28,689 & $51.8 \%$ & 9,504 & $44.6 \%$ & 9,232 & $58.8 \%$ & 9,953 & $54.2 \%$ \\
\hline Depression (n, \%) & 5,203 & $9.4 \%$ & 1,988 & $9.3 \%$ & $\mathrm{I}, 774$ & $11.3 \%$ & $|, 44|$ & $7.8 \%$ \\
\hline \# of unique Rx classes (mean, SD) & 8.06 & 6.7 & 5.79 & 5.8 & 6.59 & 6.7 & 11.96 & 5.8 \\
\hline \# of Rx (mean, SD) & 8.96 & 7.6 & 6.26 & 6.5 & 7.16 & 7.5 & 13.63 & 6.8 \\
\hline \# of unique 3-digit ICD-9 Dx codes (mean, SD) & 10.22 & 7.1 & 8.79 & 6.9 & 11.57 & 7.4 & 10.72 & 7.0 \\
\hline \multicolumn{9}{|l|}{ Reliever medication use } \\
\hline SABA canister use $(\mathrm{n}, \%)$ & 13,362 & $24.1 \%$ & I,764 & $8.3 \%$ & 2,429 & $15.5 \%$ & 9,169 & $49.9 \%$ \\
\hline OCS prescription use (n, \%) & 12,754 & $23.0 \%$ & 2,364 & $11.1 \%$ & 2,530 & $16.1 \%$ & 7,860 & $42.8 \%$ \\
\hline Nebulized IPR use $(n, \%)$ & 2,574 & $4.6 \%$ & N/A & $\mathrm{N} / \mathrm{A}$ & 482 & $3.1 \%$ & 2,092 & $11.4 \%$ \\
\hline
\end{tabular}

Abbreviations: $\mathrm{CCl}$, Charlson comorbidity index; CVD, cardiovascular disease; Dx, diagnosis; ICD-9, International Classification of Diseases, 9th Revision; IPR, ipratropium or combination ipratropium-albuterol; LRTI, lower respiratory tract infection; N/A, not applicable; OCS, oral corticosteroid; Rx, prescription; SABA, short-acting beta agonist; SD, standard deviation; URTI, upper respiratory tract infection.

\section{COPD drug use}

Almost three-fourths (74\%) of the study sample were receiving prescription medications (maintenance or reliever) for COPD, but less than half $(45 \%)$ of the study sample were receiving maintenance medications (Figure 1). Treatment rates differed substantially among the subgroups. ME patients had the highest rate of maintenance drug use (89\%) indicating that only $11 \%$ of patients previously using a maintenance medication in the pre-index year did not continue to use it in the index year. However, among the other subgroups, the uptake of maintenance medications in the index year was low, with only $19 \%$ of the MN patients and $26 \%$ of the newly diagnosed patients receiving maintenance medications in the index year. ICS + LABA was the most prevalent drug class (27\%), and this finding was consistent across all subgroups (Table 2). Both the MN and newly diagnosed subgroups were more likely to initiate maintenance therapy with ICS + LABA than any other maintenance medication including TIO. One in seven patients was receiving reliever medications, with antibiotics being the most prevalent type of reliever medication used. The use of SABAs and OCSs increased from the prior year in newly diagnosed and MN patients with only a slight increase noted for ME patients.

Patients 65 years of age and over were more likely to be treated with both maintenance medications ( $54 \%$ vs $36 \%$ ) and reliever medications ( $76 \%$ vs $62 \%$ ) compared to younger patients aged 40-64 years, and this pattern was similar across all of the subgroups (data not shown). Almost $64 \%$ of patients under the care of pulmonologists were treated with maintenance drugs compared to $40 \%$ of patients under the care of GPs (data not shown). The proportion of pulmonologist-treated patients receiving ICS + LABA or TIO was almost twice that of GPtreated patients ( $41 \%$ vs $23 \%$ and $38 \%$ vs $18 \%$, respectively). Pulmonologist-treated patients were also more likely to be receiving $\geq 2$ classes of drugs compared to GP-treated patients (37\% vs $18 \%$ ). At least $70 \%$ of both pulmonologist-treated and GP-treated patients were using a reliever medication with the rate being higher for the former ( $76 \%$ vs $70 \%$ ). 


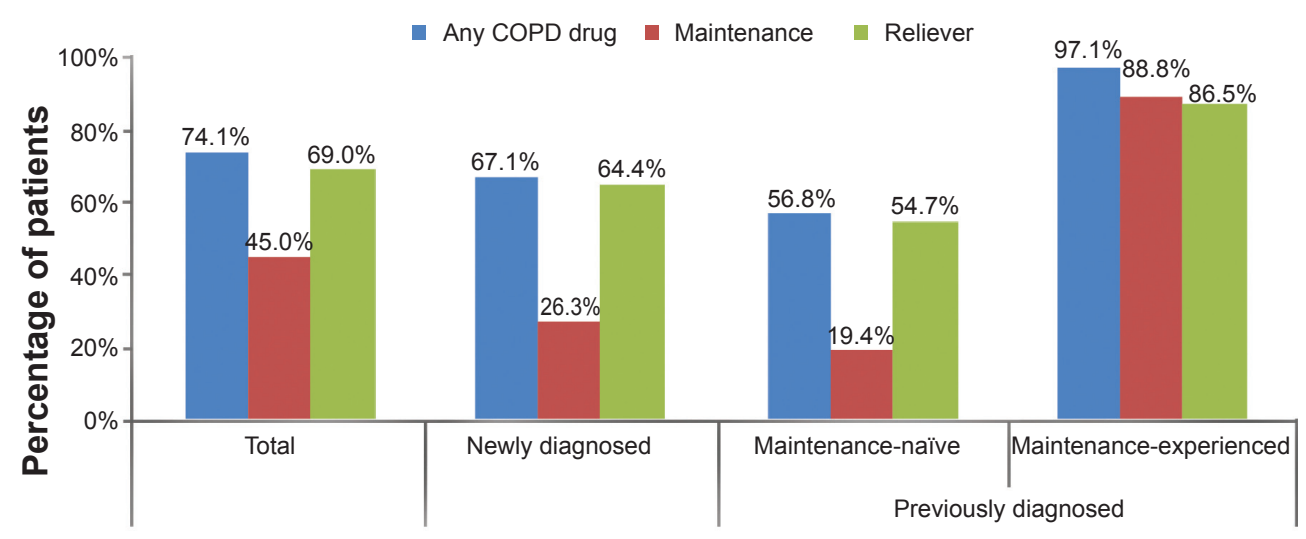

Figure I Rates of pharmacotherapy in a prevalent COPD population by prior diagnosis and maintenance-treatment status.

Abbreviation: COPD, chronic obstructive pulmonary disease.

\section{Guideline-recommended care}

In total, $28 \%$ of previously diagnosed patients had at least one exacerbation in the pre-index period. Of this subset, $36 \%$ were not using long-acting maintenance medications despite a history of exacerbations (Figure 2). More than $80 \%$ of ME patients with an exacerbation history were using long-acting maintenance medication compared to less than a quarter of MN patients with an exacerbation history. ICS-containing regimens were the most commonly used long-acting maintenance treatment in patients with an exacerbation history with $51 \%$ using ICS and/or ICS + LABA followed by TIO (38\%) (Figure 2).

A slightly higher proportion of patients $\geq 65$ years of age with an exacerbation history used long-term maintenance medications compared to patients 40-64 years old (68\% vs $60 \%$ ). However, this was only noted in ME patients, while a slightly lower proportion was observed among MN patients (data not shown). Patients under the care of pulmonologists were more likely to be on long-acting maintenance therapy compared to those who were under the care of a GP, both in the overall study sample (78\% vs 59\%) and in the $\mathrm{MN}$ and ME subgroups (data not shown). Furthermore, pulmonologist-treated patients with an exacerbation history were more likely to be using two classes of drugs compared to GP-treated patients (39\% vs 21\%).

Overall, $23 \%$ of the COPD patients experienced an acute exacerbation of COPD, which was defined by the presence of a COPD-related hospitalization or ED visit. Short-term treatment with OCS and/or antibiotics was higher for hospitalization exacerbations compared to ED visit exacerbations (Figure 3: 68\% vs 44\%). This rate differed among the subgroups with ME patients having the highest rates followed by newly diagnosed patients and $\mathrm{MN}$ patients (hospitalization: $80 \%, 66 \%$, and 50\%, and ED: 64\%, 38\%,

Table 2 Maintenance and reliever medication use patterns in COPD patients in 2008

\begin{tabular}{|c|c|c|c|c|c|c|c|c|}
\hline \multirow[b]{4}{*}{ Maintenance drug use } & \multirow{2}{*}{\multicolumn{2}{|c|}{ Total }} & \multirow{2}{*}{\multicolumn{2}{|c|}{ Newly diagnosed }} & \multicolumn{4}{|c|}{ Previously diagnosed } \\
\hline & & & & & \multirow{2}{*}{\multicolumn{2}{|c|}{$\begin{array}{l}\text { Maintenance- } \\
\text { naïve } \\
N=\mid \mathbf{5 , 6 8 8}\end{array}$}} & \multirow{2}{*}{\multicolumn{2}{|c|}{$\begin{array}{l}\text { Maintenance- } \\
\text { experienced } \\
\mathbf{N}=18,359\end{array}$}} \\
\hline & \multicolumn{2}{|c|}{$\mathbf{N}=\mathbf{5 5 , 3 6 ~ I ~}$} & \multicolumn{2}{|c|}{$\mathbf{N}=2|, 3| 4$} & & & & \\
\hline & 24,938 & $45.0 \%$ & 5,598 & $26.3 \%$ & 3,045 & $19.4 \%$ & 16,295 & $88.8 \%$ \\
\hline $\mathrm{TIO}$ & 12,248 & $22.1 \%$ & 2,232 & $10.5 \%$ & 1,238 & $7.9 \%$ & 8,778 & $47.8 \%$ \\
\hline LABA & |,7| | & $3.1 \%$ & 158 & $0.7 \%$ & 140 & $0.9 \%$ & $1,4 \mid 3$ & $7.7 \%$ \\
\hline ICS & 2,157 & $3.9 \%$ & 438 & $2.1 \%$ & 268 & $1.7 \%$ & $\mathrm{I}, 45 \mathrm{I}$ & $7.9 \%$ \\
\hline ICS + LABA & $|4,8| 4$ & $26.8 \%$ & 3,017 & $14.2 \%$ & 1,662 & $10.6 \%$ & 10,135 & $55.2 \%$ \\
\hline Inhaled IPR & 7,129 & $12.9 \%$ & 1,590 & $7.5 \%$ & 760 & $4.8 \%$ & 4,779 & $26.0 \%$ \\
\hline Xanthines & $\mathrm{I}, 704$ & $3.1 \%$ & 83 & $0.4 \%$ & 96 & $0.6 \%$ & 1,525 & $8.3 \%$ \\
\hline Reliever drug use & 38,172 & $69.0 \%$ & 13,719 & $64.4 \%$ & 8,574 & $54.7 \%$ & 15,879 & $86.5 \%$ \\
\hline SABA & 18,647 & $33.7 \%$ & 5,868 & $27.5 \%$ & 3,395 & $21.6 \%$ & 9,384 & $51.1 \%$ \\
\hline OCS & 17,457 & $31.5 \%$ & 6,017 & $28.2 \%$ & 3,331 & $21.2 \%$ & 8,109 & $44.2 \%$ \\
\hline Nebulized IPR & 3,602 & $6.5 \%$ & 776 & $3.6 \%$ & 705 & $4.5 \%$ & 2,121 & $11.6 \%$ \\
\hline$A B X$ & 33,049 & $59.7 \%$ & 12,365 & $58.0 \%$ & 7,488 & $47.7 \%$ & 13,196 & $71.9 \%$ \\
\hline
\end{tabular}

Abbreviations: $A B X$, respiratory antibiotics; COPD, chronic obstructive pulmonary disease; ICS, inhaled corticosteroid; IPR, ipratropium or combination ipratropiumalbuterol; LABA, long-acting beta agonist; OCS, oral corticosteroid; SABA, short-acting beta agonist; TIO, tiotropium. 


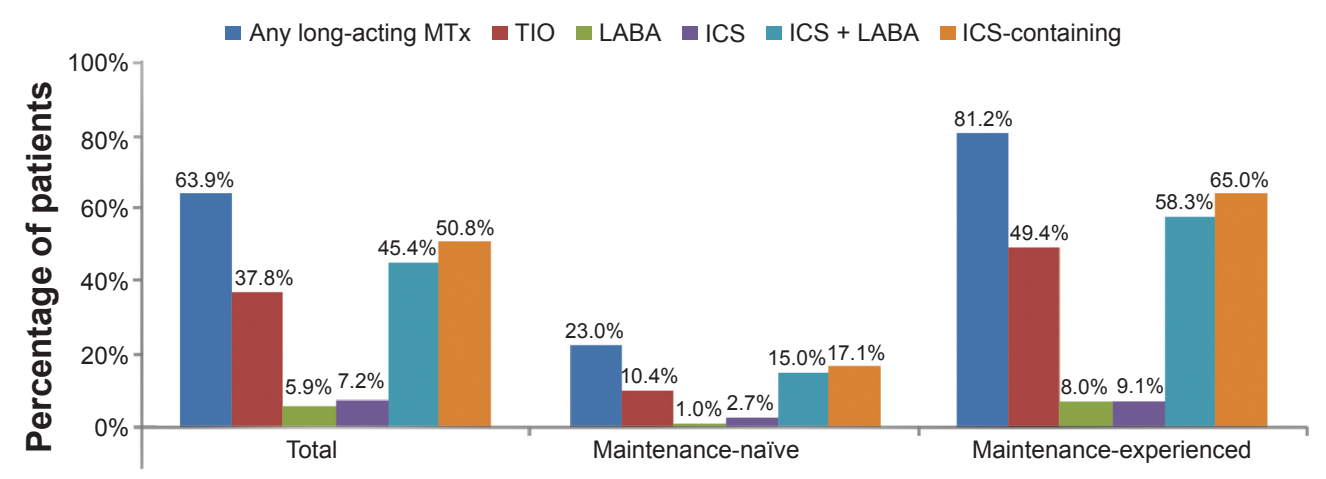

Figure 2 Prevalence of treatment with long-acting maintenance pharmacotherapy in COPD patients with an exacerbation history in 2008*.

Note: *Excludes newly diagnosed patients by definition.

Abbreviations: COPD, chronic obstructive pulmonary disease; ICS, inhaled corticosteroid; ICS + LABA, combination of inhaled corticosteroid and long-acting beta-agonist; ICS-containing, any ICS or ICS + LABA; LABA, long-acting beta agonist; MTx, maintenance treatment; TIO, tiotropium.

and $37 \%$, respectively). The type of short-term treatment did not differ by type of exacerbation. Combination therapy with an OCS and an antibiotic was the most frequently prescribed short-term treatment for acute COPD exacerbations followed by antibiotics alone and then OCS alone. This finding was consistent across all subgroups with one exception in newly diagnosed patients where antibiotics alone were most likely to be prescribed after discharge from an ED visit. Older patients ( $\geq 65$ years) were more likely to be treated with OCSs and/or antibiotics after an acute exacerbation compared to younger patients (hospitalization: $73 \%$ vs $61 \%$, and ED visit: $45 \%$ vs $42 \%$ ). Short-term treatment rates after a discharge

\section{A}

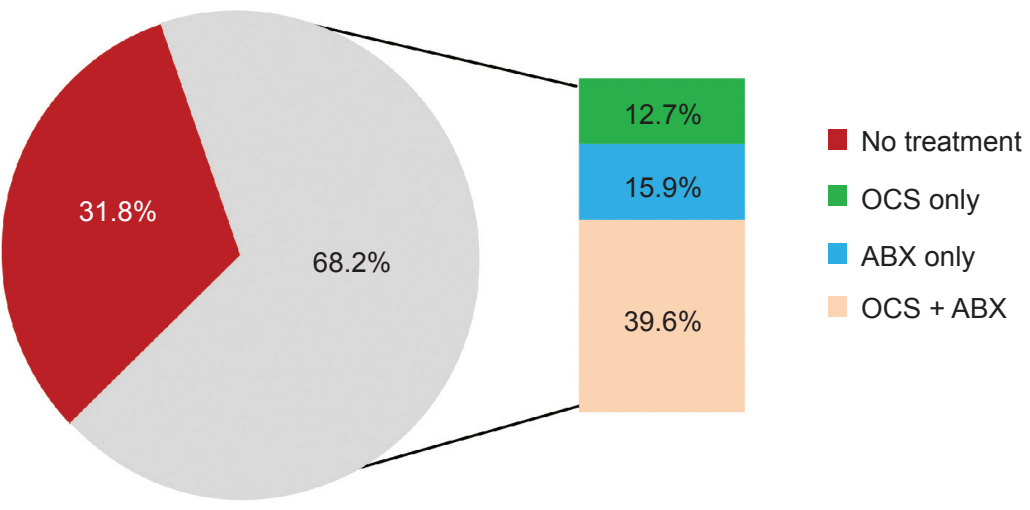

B

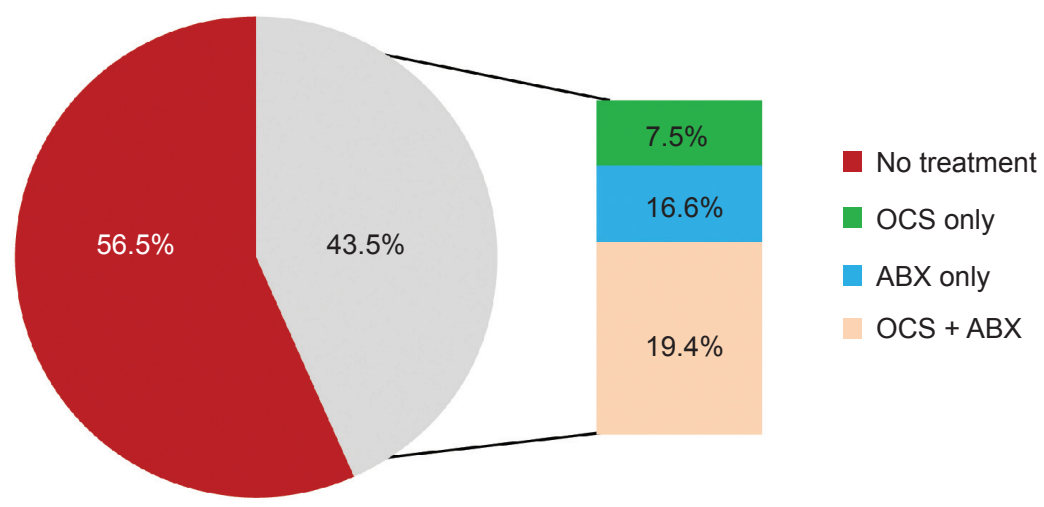

Figure 3 Short-term treatment of acute exacerbations of COPD.

Notes: (A) After discharge from COPD-related hospitalization. (B) After discharge from COPD-related ED visit.

Abbreviations: ABX, antibiotics; COPD, chronic obstructive pulmonary disease; ED, emergency department; OCS, oral corticosteroid. 
from a hospitalization exacerbation did not differ between pulmonologist- and GP-treated patients (close to 70\% for both) but were higher for pulmonologist-treated compared to GP-treated patients after an ED visit for an exacerbation (52\% vs $43 \%)$.

\section{Discussion}

In a prevalent COPD population, the present study found less than half of COPD patients to be receiving treatment with maintenance medications indicating that $55 \%$ of COPD patients are not treated with maintenance medications. In patients with at least one prior exacerbation, a third of patients $(36 \%)$ were not receiving guideline-recommended long-acting maintenance medications. Patients discharged from the ED were less likely to have short-term treatment for their acute exacerbation compared to those being discharged from the hospital (44\% vs 70\%).

Untreated rates from prior studies range from $12 \%$ to $71 \%$. ${ }^{7,8,12}$ The wide range is due to differences in the definition of treatment, with studies reporting low rates usually including reliever medications such as SABAs in their definitions. Make et al used a definition similar to our study, and they found that $66 \%$ of older patients and $59 \%$ of younger patients went untreated between 2004 and 2005. ${ }^{7}$ Our study used more recent data (2008) and found a lower untreated rate though still suboptimal (55\% overall). However, the pattern was reversed in our study between the two age groups, with older patients having a lower untreated rate compared to younger patients ( $45 \%$ vs $64 \%$ ). The prevalence of no treatment was more likely in patients who were $\mathrm{MN}$ or newly diagnosed, suggesting a low rate of initiation of maintenance medications due to either physician inertia or the possibility of patients being asymptomatic and not requiring treatment.

However, even when considering patients for whom maintenance therapy may be most appropriate, eg, patients with prior exacerbations, a suboptimal maintenance therapy rate of $64 \%$ was observed. The results were similar, albeit slightly higher $(71 \%)$, when the definition of patients with prior exacerbations was modified as those with at least two compared to at least one exacerbation to align with changes in the GOLD guidelines since the study was conducted. Appropriate care was evaluated similarly in three studies, ${ }^{7-9}$ and all three have consistently reported suboptimal treatment rates in patients for whom treatment would be recommended (eg, patients with prior exacerbations or patients with high severity). It is notable that consistent with the overall finding, the low rate of treatment in the subset of patients with an exacerbation history was primarily driven by patients who were naïve to maintenance medications. This consistent finding suggests that improvement in COPD patient care is imperative. Despite the advent of multiple pharmacotherapy regimens in the last decade, prevention of COPD exacerbations and treating patients optimally post-exacerbations still present an unmet need.

Several studies have reported short-term treatment with OCSs for an exacerbation requiring a hospitalization or ED visit with rates ranging from $51 \%$ to $90 \%$. The wide variation is likely to be related to the period of assessment. ${ }^{7,13-15}$ Lower rates were found in studies that evaluated treatment $3-5$ days after discharge $(51 \%-55 \%),{ }^{7,13}$ and higher rates were found in studies that evaluated treatment during the exacerbation visit (85\%-90\%). ${ }^{14,15}$ Our study evaluated treatment after discharge and found similar rates to those reported in the literature.

The study findings need to be interpreted in light of the study limitations. The appropriate use of COPD pharmacotherapy could not be fully evaluated based on severity of disease due to the lack of clinical information as well as access to physician prescribing decisions or factors used in making those decisions. However, the study attempted to evaluate the use of long-acting maintenance drug in COPD patients who had an exacerbation history (ie, in patients for whom long-acting maintenance treatment would be recommended by guidelines). The rates of short-term treatment after discharge from acute exacerbation visits were based on prescriptions dispensed post-discharge and not those received during the acute exacerbation visit, and hence may likely be underestimated. This dataset presents the caveat of generalizability since it represents a managed care population that may be different from other populations.

In conclusion, the results indicate a prevalence of undertreatment, although there seems to be a slight improvement compared to prior years. This improvement may reflect the impact of landmark studies and newer classes of maintenance medications demonstrating benefits of newer maintenance therapy in COPD. ${ }^{10,11}$ In terms of guideline-recommended care, this study has found similar rates of treatment compared to prior years, which suggests that gaps in adherence to guidelines still exist. COPD patient care for exacerbations still requires improvement. Future research should evaluate the impact of adherence to guidelines on outcomes.

\section{Acknowledgment}

The authors would like to acknowledge Manan Shah for the critical review of the study protocol during his employment at Xcenda. 


\section{Disclosure}

Funding for this study was provided by GlaxoSmithKline (GSK). GBD is a professor at John Hopkins University and received payment from GSK for participation in this project. AAD and SPN are employees of GSK and own company stock. AOD and OEL are employees of Xcenda, LLC, a company that received funding from GSK to conduct this research. The authors report no other conflicts of interest in this work.

\section{References}

1. Global Initiative for Chronic Obstructive Lung Disease (GOLD). Global Strategy for the Diagnosis, Management, and Prevention of Chronic Obstructive Pulmonary Disease; 2013. Available from: http://www. goldcopd.org. Accessed August 15, 2013.

2. The National Heart, Lung, and Blood Institute. Lung Diseases InformationWhat is COPD?; 2013. Available from: http://www.nhlbi.nih.gov/health/ health-topics/topics/copd. Accessed August 15, 2013.

3. Miniño AM, Xu J, Kochanek KD. Deaths: final data for 2008. Natl Vital Stat Rep. 2011;59(10):1-126.

4. Anthonisen NR, Connett JE, Kiley JP, et al. Effects of smoking intervention and the use of an inhaled anticholinergic bronchodilator on the rate of decline of FEV1. The Lung Health Study. JAMA. 1994;272(19): 1497-1505.

5. Camilli AE, Burrows B, Knudson RJ, Lyle SK, Lebowitz MD. Longitudinal changes in forced expiratory volume in one second in adults. Effects of smoking and smoking cessation. Am Rev Respir Dis. 1987; 135(4):794-799.

6. Diette GB, Orr P, McCormack MC, Gandy W, Hamar B. Is pharmacologic care of chronic obstructive pulmonary disease consistent with the guidelines? Popul Health Manag. 2010;12(1):21-26.
7. Make B, Dutro MP, Paulose-Ram R, Marton JP, Mapel DW. Undertreatment of COPD: a retrospective analysis of US managed care and medicare patients. Int J Chron Obstruct Pulmon Dis. 2012;7:1-9.

8. Asche CV, Leader S, Plauschinat C, et al. Adherence to current guidelines for chronic obstructive pulmonary disease (COPD) among patients treated with combination of long-acting bronchodilators or inhaled corticosteroids. Int J Chron Obstruct Pulmon Dis. 2012;7:201-209.

9. Tashkin DP, Celli B, Senn S, et al; UPLIFT Study Investigators. A 4-year trial of tiotropium in chronic obstructive pulmonary disease. N Engl J Med. 2008;359(15):1543-1554.

10. Calverley PM, Anderson JA, Celli B, et al; TORCH investigators. Salmeterol and fluticasone propionate and survival in chronic obstructive pulmonary disease. N Engl J Med. 2007;356(8):775-789.

11. Lee TA, Wilke C, Joo M, et al. Outcomes Associated with Tiotropium use in Chronic Obstructive Pulmonary Disease Patients. Effective Health Care Research Report No 15. (Prepared by Chicago-Area DEcIDE Center Under Contract No. HSA29020050038I TO4). Rockville, MD: Agency for Healthcare Research and Quality; 2009. Available from: http://effectivehealthcare.ahrq.gov/reports/final.cfm. Accessed August 15, 2013.

12. Feifer RA, Aubert R, Verbrugge RR, Khalid M. Disease management opportunities for chronic obstructive pulmonary disease: gaps between guidelines and current practice. Dis Manag. 2002;5(3):143-156.

13. Cydulka RK, Rowe BH, Clark S, Emerman CL, Camargo CA Jr; MARC Investigators. Emergency department management of acute exacerbations of chronic obstructive pulmonary disease in the elderly: the multicenter airway research collaboration. $J$ Am Geriatr Soc. 2003;51(7):908-916.

14. Dalal AA, Shah M, D'Souza AO, Rane P. Costs of COPD exacerbations in the emergency department and inpatient setting. Respir Med. 2011;105(3):454-460.

15. Lindenauer PK, Pekow P, Gao S, Crawford AS, Gutierrez B, Benjamin EM. Quality of care for patients hospitalized for acute exacerbations of chronic obstructive pulmonary disease. Ann Intern Med. 2006;144(12):894-903.
International Journal of COPD

\section{Publish your work in this journal}

The International Journal of COPD is an international, peer-reviewed journal of therapeutics and pharmacology focusing on concise rapid reporting of clinical studies and reviews in COPD. Special focus is given to the pathophysiological processes underlying the disease, intervention programs, patient focused education, and self management protocols.

\section{Dovepress}

This journal is indexed on PubMed Central, MedLine and CAS. The manuscript management system is completely online and includes a very quick and fair peer-review system, which is all easy to use. Visit $\mathrm{http}: / / \mathrm{www}$.dovepress.com/testimonials.php to read real quotes from published authors. 